\title{
MicroRNA-15b participates in the development of peripheral arterial disease by modulating the growth of vascular smooth muscle cells
}

\author{
YONG SUN, YONG GAO, TAO SONG, CHAOWEN YU, ZHONGLIN NIE and XIAOGAO WANG
}

Department of Vascular Surgery, The First Affiliated Hospital of Bengbu Medical College, Bengbu, Anhui 233004, P.R. China

Received July 16, 2018; Accepted February 6, 2019

DOI: $10.3892 /$ etm.2019.7552

\begin{abstract}
As an atherosclerotic disease, the process of peripheral arterial disease (PAD) is complicated and includes the abnormal proliferation of vascular smooth muscle. The current study aimed to determine the role of microRNA-15b (miR-15b) in the development of PAD and its associated mechanisms. Human vascular smooth muscle cells (hVSMCs) were used in the current study. To assess the effects of miR-15b on hVSMCs, miR-15b was up- or downregulated in hVSMCs using miR-15b mimics or miR-15b inhibitors respectively. Cell viability, migration and apoptosis were then determined via MTT, transwell and flow cytometry assays, respectively. TargetScan bioinformatics software was utilized to predict the targets of miR-15b, and the binding sites between insulin growth factor 1 receptor (IGF1R) and miR-15b were confirmed by dual-luciferase reporter assay. The results reveled that the miR-15b mimic significantly reduced hVSMC cell viability and migration, and promoted cell apoptosis. However, the opposite effect was observed following miR-15b inhibitor transfection. It was also determined that miR-15b directly targeted IGF1R and negatively regulated its expression in hVSMCs. Additionally, the results demonstrated that the miR-15b mimic inhibited the $\mathrm{PI} 3 \mathrm{~K} / \mathrm{AKT}$ signaling pathway in hVSMCs, whereas the miR-15b inhibitor promoted it. Furthermore, the results indicated that the effect of the miR-15b mimic on hVSMCs was reversed by IGF1R overexpression. In conclusion, the data indicated that miR-15b participated in the occurrence and development of PAD by modulating hVSMC proliferation, apoptosis and migration via the regulation of IGF1R expression.
\end{abstract}

\section{Introduction}

Peripheral arterial disease (PAD) is caused by limb artery atherosclerosis (1), which is most common in the lower

Correspondence to: Dr Tao Song, Department of Vascular Surgery, The First Affiliated Hospital of Bengbu Medical College, 287 Changhuai Road, Bengbu, Anhui 233004, P.R. China

E-mail: songtao201807@163.com

Key words: peripheral arterial disease, human vascular smooth muscle cells, microRNA-15b, insulin-like growth factor 1 receptor extremities (2). PAD affects $\sim 20 \%$ of the population aged $>55$ years, worldwide (3). The Inter-Society Consensus for the Management of Peripheral Arterial Disease (TASC II) determined that the prevalence of asymptomatic PAD is $\sim 3-10 \%$ and is increasing to $15-20 \%$ in people aged $>70$ years worldwide (4). Critical limb ischemia (CLI) is the most severe clinical manifestation of PAD and may cause intermittent claudication, gangrene and foot ulcers (5). Patients with CLI therefore have a risk of destructive complications, including amputation and mortality (6,7). Smoking and diabetes are major risk factors for PAD, in addition to arterial hypertension, hypercholesterolemia, familial susceptibility and the male sex (8). Prevalence is also particularly age-associated, as $20 \%$ of individuals over the age of 70 are affected (9). The pathophysiological mechanism of PAD is complex and has not yet been fully elucidated (1). Despite major improvements in surgical endovascular techniques (1), there is currently no therapy that can effectively treat and improve the prognosis of patients with severe PAD (10). Thus, PAD still has a high mortality and morbidity (9). Therefore, identifying the molecular mechanism underlying PAD development is critical for determining novel treatments. In recent years, the cellular and molecular mechanisms of PAD (10) and the role of certain microRNAs (miRNAs) in PAD have received increasing attention (11).

miRNAs are small non-coding RNAs that are 22 nucleotide in length, which post-transcriptionally regulate gene expression by degrading mRNAs or inhibiting protein translation (4). As key regulators of certain events, miRNAs serve important roles in the regulation of the balance between cell proliferation and differentiation during tumorigenesis and organ development (12). Previous studies have demonstrated that miRNAs serve critical roles in the regulation of vascular cell proliferation, differentiation and apoptosis (13-15). However, the biological function of miRNAs in PAD has only recently been elucidated. miRNAs have been reported to serve critical roles in PAD and PAD-associated complications (13-15). However, the role of miR-15b in PAD is still unclear.

The present study aimed to assess the role of miR-15b in the development of PAD and its associated mechanisms.

\section{Materials and methods}

Cell culture. Human vascular smooth muscle cells (hVSMCs) were obtained from the Cell Bank of the Shanghai Institute 
of Cell Biology (Shanghai, China). Cells were grown in $75 \mathrm{~cm}^{2}$ flasks with Dulbecco's Modified Eagle's medium (Gibco; Thermo Fisher Scientific, Inc., Waltham, MA, USA) containing $10 \%$ fetal bovine serum (FBS; Gibco; Thermo Fisher Scientific, Inc.), $100 \mathrm{U} / \mathrm{ml}$ penicillin and $100 \mu \mathrm{g} / \mathrm{ml}$ streptomycin (Beyotime Institute of Biotechnology, Shanghai, China). Cells were then incubated at $37^{\circ} \mathrm{C}$ with $5 \% \mathrm{CO}_{2}$.

Cell transfection. $100 \mathrm{nM}$ Mimic controls (sense: 5'-UUC UCCGAACGUGUCACGUTT-3'; anti-sense: 5-'ACGUGA CACGUUCGGAGAATT-3'), $100 \mathrm{nM}$ miR-15b mimics (sense: 5'-UAGCAGCACAUCAUGGUUUACA-3'; anti-sense: 5'-UAA ACCAUGAUGUGCUGCUAUU-3'), 100 nM miR-15b inhibitors (5'-UGUAAACCAUGAUGUGCUGCUA-3'), $100 \mathrm{nM}$ inhibitor controls (5'-CAGUACUUUUGUGUAGUACAA-3'; all Shanghai GenePharma Co., Ltd., Shanghai, China), $2 \mu 1$ control-plasmids (cat. no. sc-108083), $2 \mu \mathrm{l}$ insulin growth factor 1 receptor (IGF1R)-plasmids (cat. no. sc-421057-ACT; both Santa Cruz Biotechnology, Inc., Santa Cruz, CA, USA) and $100 \mathrm{nM}$ miR-15b mimics+2 $\mu \mathrm{l}$ IGF1R-plasmids were transfected into hVSMCs using Lipofectamine ${ }^{\circledR} 2000$ (Thermo Fisher Scientific, Inc.) in accordance with the manufacturer's protocol. Cells without any treatment were used as the control group. Following $48 \mathrm{~h}$, reverse transcription-quantitative polymerase chain reaction (RT-qPCR) was performed to assess transfection efficiency.

MTT assay. A MTT assay was performed to detect cell viability. $48 \mathrm{~h}$ following transfection, cells were seeded in 96-well plates $\left(2 \times 10^{4}\right.$ cells $\left./ \mathrm{ml}\right)$. Subsequently, $10 \mu \mathrm{l}$ MTT reagent (Beyotime Institute of Biotechnology, Haimen, China) was added to each well and incubated for $4 \mathrm{~h}$ at $37^{\circ} \mathrm{C}$. DMSO (100 $\mu$ l; Nanjing KeyGen Biotech Co., Ltd., Nanjing, China) was added to dissolve the formazan crystals. Thermo Scientific $^{\mathrm{TM}}$ Multiskan $^{\mathrm{TM}}$ FC (Thermo Fisher Scientific, Inc.) was then used to measure absorbance at a wavelength of $490 \mathrm{~nm}$. Each experiment was performed in triplicate.

Cell apoptosis assay. Following transfection for $48 \mathrm{~h}$, hVSMCs were digested using $0.2 \%$ trypsin. Following a wash with PBS, cells were fixed with $70 \%$ ethanol overnight at $4^{\circ} \mathrm{C}$. The apoptosis of cells was then assessed using the Annexin V-fluorescein isothiocyanate/propidium iodide apoptosis detection kit [cat. no. 70-AP101-100; Hangzhou Multi Sciences (Lianke) Biotech Co., Ltd., Hangzhou, China] following the manufacturer's protocol. A FACS Calibur flow cytometer with Cell Quest software (version 5.1; BD Biosciences, San Jose, CA, USA) was utilized for the detection of cell apoptosis rate. Each experiment was performed in triplicate.

Transwell assay. To assess cell migration, un-coated transwell chambers (pore size, $8 \mu \mathrm{m}$; Costar; Corning Inc., Corning, NY, USA) were utilized in the present study. Cells $\left(2 \times 10^{4}\right)$ were seeded into the upper chamber with serum-free DMEM and $600 \mu \mathrm{l}$ DMEM containing 30\% FBS was added to the lower chamber. Following $48 \mathrm{~h}$ of incubation at $37^{\circ} \mathrm{C}$, the migratory cells on the lower chamber were fixed with $4 \%$ paraformaldehyde at room temperature for $30 \mathrm{~min}$ and then stained with $0.5 \mathrm{ml} 0.1 \%$ crystal violet at room temperature for $15 \mathrm{~min}$. At the end of the experiment, migrated cells were counted under a light microscope at a magnification of x200 using five random fields of view. Each experiment was performed in triplicate.

Dual luciferase reporter assay. TargetScan bioinformatics software (www.targetscan.org/vert_71) was utilized to predict the targets of miR-15b and the binding sites between IGF1R and miR-15b. To confirm the binding sites between miR-15b and the 3'-untranslated region (3'-UTR) of IGF1R, a dual luciferase reporter assay was performed. The wild type (WT-IGF1R) and mutant (MUT-IGF1R) 3'-UTRs of IGF1R were cloned into a pmiR-RB-ReportTM dual luciferase reporter gene plasmid vector (Guangzhou RiboBio Co., Ltd., Guangzhou, China). hVSMCs were then co-transfected with WT-IGF1R or MUT-IGF1R with miR-15b mimics or mimic controls using Lipofectamine ${ }^{\circledR} 2000$ (Invitrogen; Thermo Fisher Scientific, Inc.) following the manufacturer's protocol. After cell transfection for $48 \mathrm{~h}$, the dual-luciferase assay system (Promega Corporation, Madison, WI, USA) was utilized to detect luciferase activity. Luciferase activity was normalized to that of renilla luciferase in the current study.

$R T$-qPCR. The TRIzol reagent (Invitrogen; Thermo Fisher Scientific, Inc.) was used for hVSMC RNA extraction and the miScript Reverse Transcription kit (Qiagen $\mathrm{GmbH}$, Hilden, Germany) was used for reverse transcription. The QuantiFast SYBR Green PCR kit (Qiagen $\mathrm{GmbH}$ ) was used to perform RT-qPCR analysis under a CFX Connect Real-Time System (Bio-Rad Laboratories, Inc., Hercules, CA, USA). GAPDH and U6 were utilized as mRNA and miRNA controls, respectively. Primer sequences as shown in Table I. The following thermocycling conditions were used for the qPCR: Initial denaturation at $95^{\circ} \mathrm{C}$ for $10 \mathrm{~min}, 35$ cycles of $95^{\circ} \mathrm{C}$ for $15 \mathrm{sec}$ and $55^{\circ} \mathrm{C}$ for $40 \mathrm{sec}$. Relative gene expression was calculated using the $2^{-\Delta \Delta \mathrm{Cq}}$ method (16).

Western blotting. Protein was extracted from cells using radioimmunoprecipitate lysate buffer containing PMSF (Beyotime Institute of Biotechnology). Total protein was quantified using a bicinchoninic acid assay kit (Pierce; Thermo Fisher Scientific, Inc.). Protein samples (70 $\mathrm{mg}$ of each extract) were separated using $12 \%$ SDS-PAGE, electrotansfered to polyvinylidene difluoride membranes and then blocked with 5\% skimmed milk at room temperature for $1.5 \mathrm{~h}$. Subsequently, membranes were incubated with the following primary antibodies overnight at $4^{\circ} \mathrm{C}$ : anti-IGF1R (1:1,000; cat. no. sc-81464; Santa Cruz Biotechnology, Inc., Dallas, TX, USA), anti-protein kinase B (AKT; 1:1,000; cat. no. 4691; Cell Signaling Technology Inc., Danvers, MA, USA), anti-phosphorylated (p)-AKT (1:1,000; cat. no. 4060; Cell Signaling Technology Inc.), anti-mechanistic target of rapamycin (mTOR; 1:1,000; cat. no. 2983; Cell Signaling Technology Inc.), anti-p-mTOR (1:1,000; cat. no. 5536; Cell Signaling Technology Inc.), anti-ribosomal protein S6 kinase beta-1 (p70S6K; 1:1,000; cat. no. 2708; Cell Signaling Technology Inc.), anti-p-P70S6K (1:1,000; cat. no. 9234; Cell Signaling Technology Inc.) and anti- $\beta$-actin (1:1,000; cat. no. 4970; Cell Signaling Technology Inc.). Samples were then incubated with horseradish peroxidase-conjugated anti-rabbit IgG secondary antibodies (1:1,000; cat. no. 7074; Cell Signaling Technology Inc.) at room temperature for $2 \mathrm{~h}$. To visualize immunoreactive proteins, the enhanced 
Table I. Primer sequences for PCR.

\begin{tabular}{lll}
\hline Gene & Direction & \multicolumn{1}{c}{ Sequences $\left(5^{\prime}-3^{\prime}\right)$} \\
\hline miR-15b & $\mathrm{F}$ & GTCGTATCCAGTGCAGGGTCCGAGGTATTCGCACTGGATACGACTGTAAA \\
IGF1R & $\mathrm{R}$ & ACGTAGCAGCACATCATGGTTT \\
& $\mathrm{F}$ & CCCCCTCGAGGATCCTGAATCTGTGCAAAC \\
AKT & $\mathrm{R}$ & AAAAGCGGCCGCCTTCCCAGCGAAATCATC \\
& $\mathrm{F}$ & TAAAGAAGGAGGTCATCGTGG \\
mTOR & $\mathrm{R}$ & CGGGACAGGTGGAAGAAAA \\
& $\mathrm{F}$ & ATGCTGTCCCTGGTCCTTATG \\
P70S6K & $\mathrm{R}$ & GGGTCAGAGAGTGGCCTTCAA \\
& $\mathrm{F}$ & AGTAAAGCATCCCTTCATCGTGG \\
GAPDH & $\mathrm{R}$ & TGATGTAAATGCCCCAAAGCC \\
& $\mathrm{F}$ & CTTTGGTATCGTGGAAGGACTC \\
U6 & $\mathrm{R}$ & GTAGAGGCAGGGATGATGTTCT \\
& $\mathrm{F}$ & GCTTCGGCAGCACATATACTAAAAT \\
& $\mathrm{R}$ & CGCTTCACGAATTTGCGTGTCAT
\end{tabular}

F, forward; R, reverse; miR, microRNA; IGF, insulin growth factor 1 receptor; P70S6K, ribosomal protein S6 kinase beta-1.

chemiluminescence detection system (Thermo Fisher Scientific, Inc.) was utilized. Gel-Pro Analyzer densitometry software (Version 6.3, Media Cybernetics, Inc., Rockville, MD, USA) was used for band density quantification.

Statistical analysis. Statistical analyses were performed using SPSS 18.0 (SPSS, Inc., Chicago, IL, USA). Data were expressed as the mean \pm standard deviation. Comparisons between two groups were made using Student's t-test and comparisons between multiple groups were analyzed by one-way analysis of variance with a Tukey's post-hoc test. $\mathrm{P}<0.05$ was considered to indicate a statistically significant difference.

\section{Results}

miR-15b affects the viability and migration of hVSMCs. To assess the role of miR-15b in the viability and migration of hVSMCs, miR-15b mimics, mimic controls, miR-15b inhibitors or inhibitor controls were transfected into hVSMCs. A total of $48 \mathrm{~h}$ following cell transfection, RT-qPCR was performed to detect transfection efficiency. The results revealed that, compared with the control group, the miR-15b mimic significantly increased the level of miR-15b, while the miR-15b inhibitor significantly decreased miR-15b (Fig. 1A). Subsequently, MTT and transwell assays were performed to determine whether miR-15b effected viability and migration of hVSMC cells. It was demonstrated that, compared with the control group, the miR-15b mimic significantly reduced the viability and migration of hVSMCs, while the miR-15b inhibitor exhibited the opposite effect (Fig. 1B and C).

miR-15b affects the apoptosis of hVSMCs. The current study further assessed whether miR-15b affected the proliferation of $\mathrm{hVSMCs}$ by inducing cell apoptosis. A total of $48 \mathrm{~h}$ following transfection, the apoptosis of hVSMCs was analyzed via flow cytometry. The results revealed that, compared with the control group, the miR-15b mimic significantly promoted the apoptosis of hVSMCs, while the miR-15b inhibitor reduced hVSMCs apoptosis (Fig. 2).

miR-15b directly targets IGF1R in hVSMCs. To assess the molecular mechanism of miR-15b on hVSMCs, bioinformatics software (TargetScan) was used to predict the potential targets of miR-15b (Fig. 3A). A dual luciferase reporter assay was then performed to confirm predictions. The results indicated that, compared with cells co-transfected with WT-IGF1R and mimic control, the luciferase activity in cells co-transfected with WT-IGF1R and miR-15b mimic were significantly reduced (Fig. 3B). The results demonstrated that IGF1R is a direct target of miR-15b.

Furthermore, the current study revealed that, compared with the control group, the miR-15b mimic significantly inhibited the expression of IGF1R in hVSMCs at the mRNA and protein level, while the miR-15b inhibitor exhibited the opposite effects (Fig. 3C and D).

miR-15b affects the phosphoinositide 3-kinase (PI3K)/AKT signaling pathway in hVSMCs. The PI3K/AKT signaling pathway, an IGF1R-mediated downstream signaling pathway, was assessed in the current study. The results indicated that, compared with the control group, the mRNA levels of key components of the PI3K/AKT pathway, including AKT, mTOR and P70S6K, were significantly decreased in hVSMCs transfected with miR-15b mimics Additionally, treatment with the miR-15b inhibitor exhibited the opposite effect (Fig. 4A-C). Compared with the control group, the protein levels of AKT/p-AKT, mTOR/p-mTOR and P70S6K/p-P70S6K in hVSMCs were significantly inhibited by miR-15b mimics and significantly enhanced by miR-15b inhibitors (Fig. 4D-G).

miR-15b mimics inhibit cell viability and, migration, and induce apoptosis in hVSMCs by targeting IGF1R. To assess 

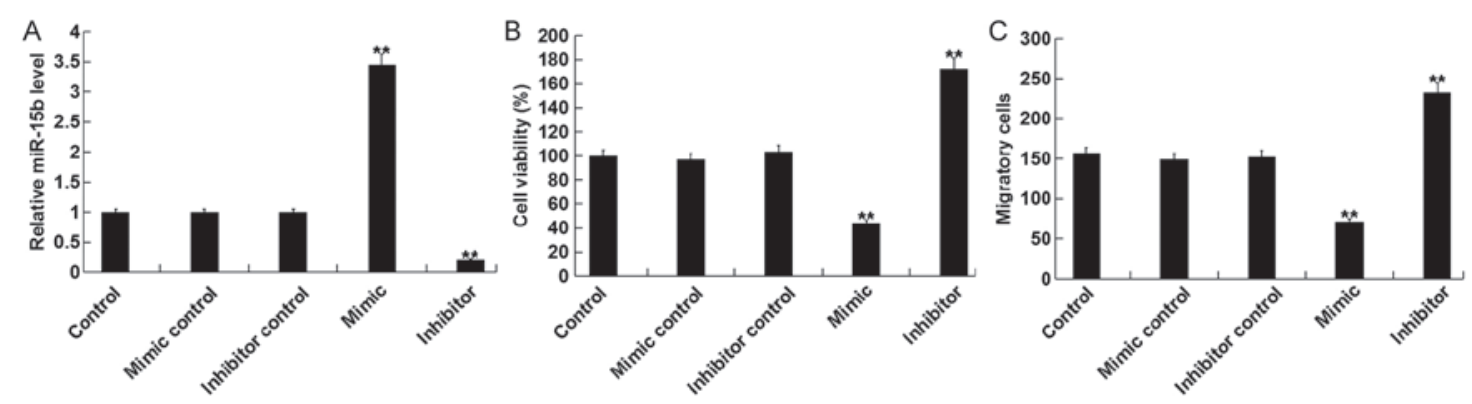

Figure 1. Effect of miR-15b on hVSMCs viability and migration. Mimic controls, miR-15b mimics, miR-15b inhibitors or inhibitor controls were transfected into hVSMCs for $48 \mathrm{~h}$. (A) Relative miR-15b levels in hVSMCs from different groups, (B) The viability and (C) migration of hVSMCs was determined via MTT and transwell assays, respectively. All data were presented as the mean \pm standard deviation. ${ }^{* *} \mathrm{P}<0.01 \mathrm{vs.}$. the control. miR, microRNA; hVSMCs, human vascular smooth muscle cells.
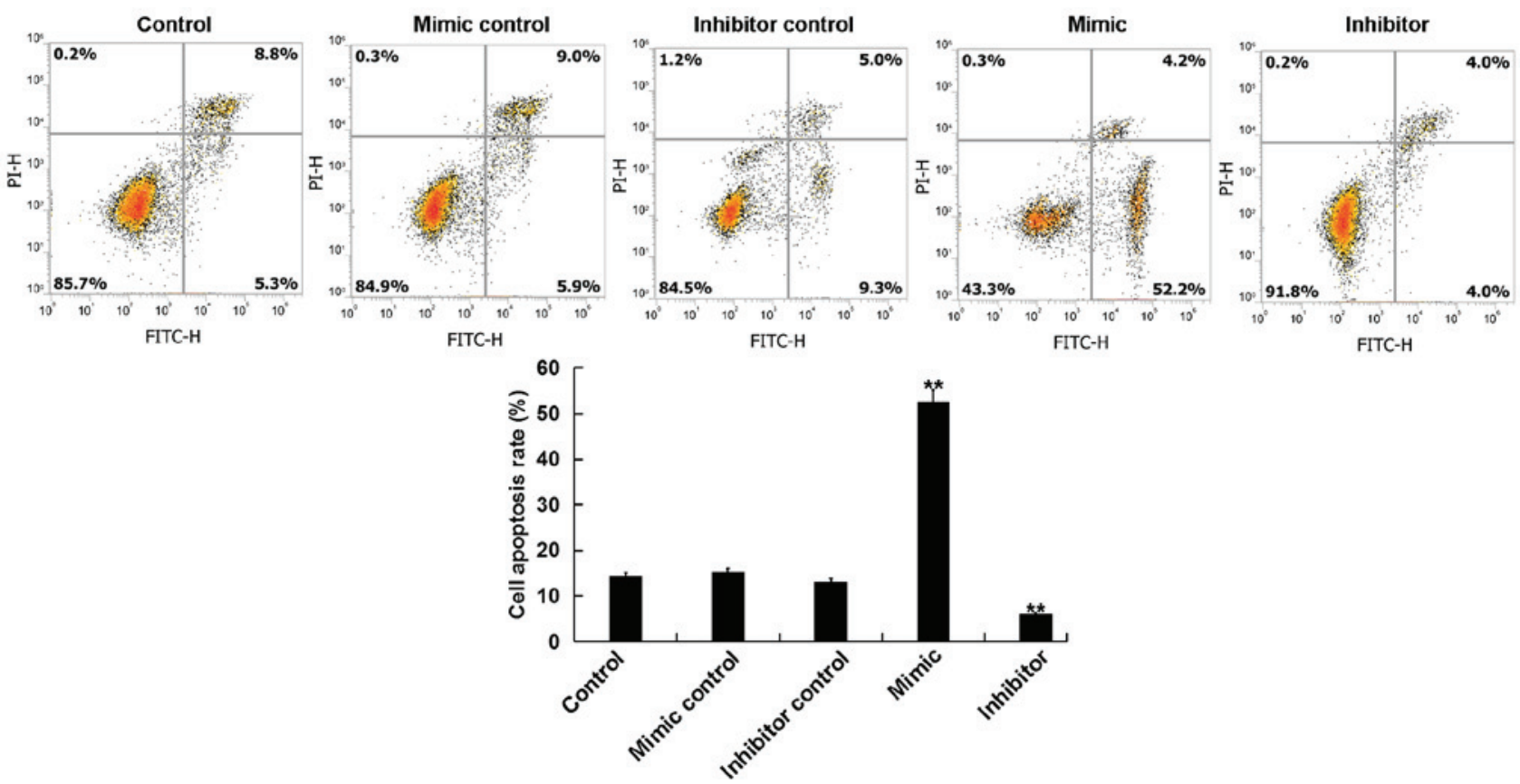

Figure 2. Effect of miR-15b on hVSMC apoptosis. Mimic controls, miR-15b mimics, miR-15b inhibitors or inhibitor controls were transfected into hVSMCs for $48 \mathrm{~h}$. Cell apoptosis rate was subsequently analyzed using flow cytometry. All data are presented as the mean \pm standard deviation. ${ }^{* *} \mathrm{P}<0.01$ vs. the control. miR, microRNA; hVSMCs, human vascular smooth muscle cells; PI, propidium iodide; FITC, fluorescein isothiocyanate.

whether miR-15b affects hVSMCs by directly acting on IGF1R, a rescue experiment was performed. Mimic controls, miR-15b mimics, control-plasmids, IGF1R-plasmids or miR-15b mimic+IGF1R-plasmids were transfected into hVSMCs. A total of $48 \mathrm{~h}$ following, transfection efficiency was detected via RT-qPCR and western blotting. As presented in Fig. 5A and B, compared with the control group, the IGF1R-plasmid significantly promoted the protein and mRNA expression of IGF1R in hVSMCs. Furthermore, compared with the mimic control group, miR-15b mimics markedly reduced the protein and mRNA expression of IGF1R in hVSMCs, while the IGF1R-plasmid reversed this effect (Fig. 5C and D).

hVSMC viability, migration and apoptosis were measured using MTT, transwell and flow cytometry assays, respectively. The results revealed that, compared with the mimic group, the reduced viability and migration, and the increased apoptosis of hVSMCs induced by the miR-15b mimic was markedly reversed following transfection with the IGF1R-plasmid (Fig. 6A-D).

\section{Discussion}

The results of the present study indicated that miR- $15 \mathrm{~b}$ overexpression significantly inhibited cell viability and cell migration, induced apoptosis and repressed the PI3K/AKT signaling pathway in hVSMCs. Furthermore, miR-15b downregulation exhibited the opposite effect. IGF1R was also identified as a direct target of miR-15b, which was negatively regulated by miR-15b. In addition, the effect of miR-15b upregulation on hVSMCs was eliminated by IGF1R upregulation. Therefore, these data indicated that miR-15b inhibited the growth of hVSMCs by directly targeting IGF1R. miR-15b may therefore be a novel therapeutic target for future PAD treatment.

PAD is a leading cause of mortality worldwide that is associated with widespread vascular atherosclerosis, affecting vessels including the coronary arteries (9). It is considered to be a clinical manifestation of systemic atherosclerosis (17). Although a large number of studies have assessed the 


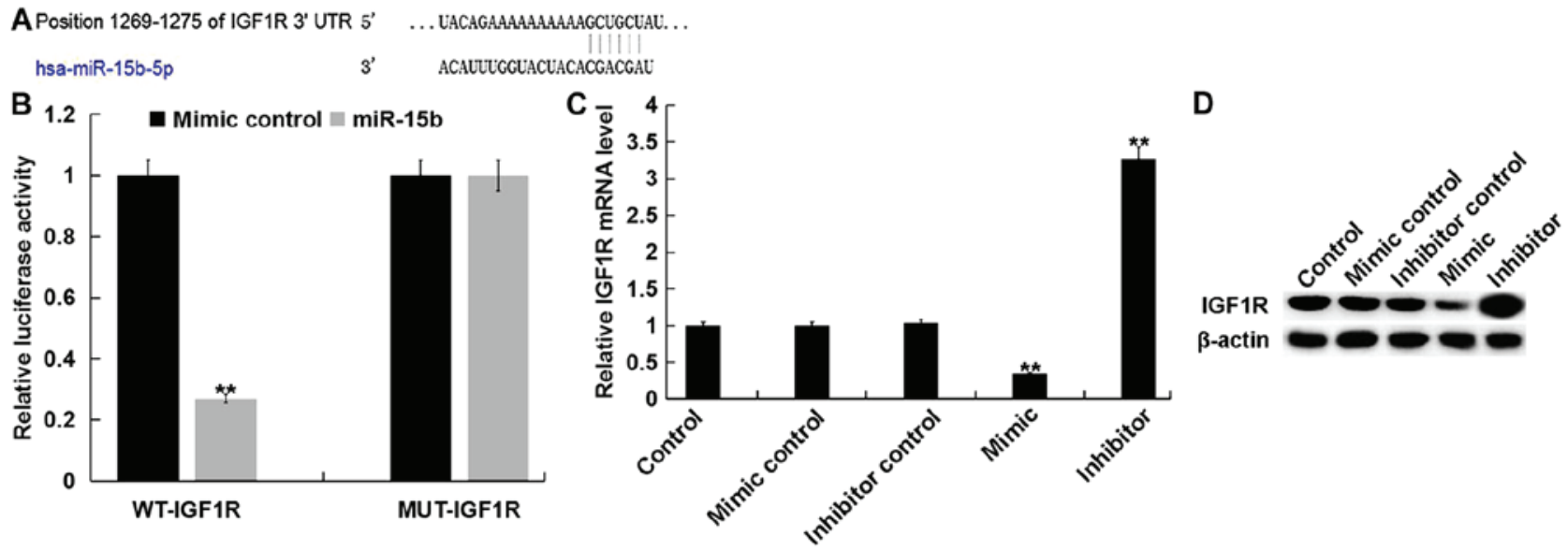

Figure 3. IGF1R is a target of miR-15b. (A) Binding sites between miR-15b and the 3'UTR of IGF1R were predicted via TargetScan software. (B) The luciferase activity of reporters containing the WT-IGF1R 3'UTR or MUT-IGF1R 3'UTR are presented. ${ }^{* *} \mathrm{P}<0.01$ vs. the mimic control. Relative (C) mRNA and (D) protein levels of IGF1R in hVSMCs were determined via reverse transcription-quantitative polymerase chain reaction and western blotting, respectively. Tests were performed $48 \mathrm{~h}$ following transfection with miR-15b mimics, mimic controls, miR-15b inhibitors or inhibitor controls. All data are presented as the mean \pm standard deviation. ${ }^{* *} \mathrm{P}<0.01$ vs. the control. IGF1R, insulin growth factor 1 receptor; miR, microRNA; UTR, untranslated region; WT, wild-type; MUT, mutant.
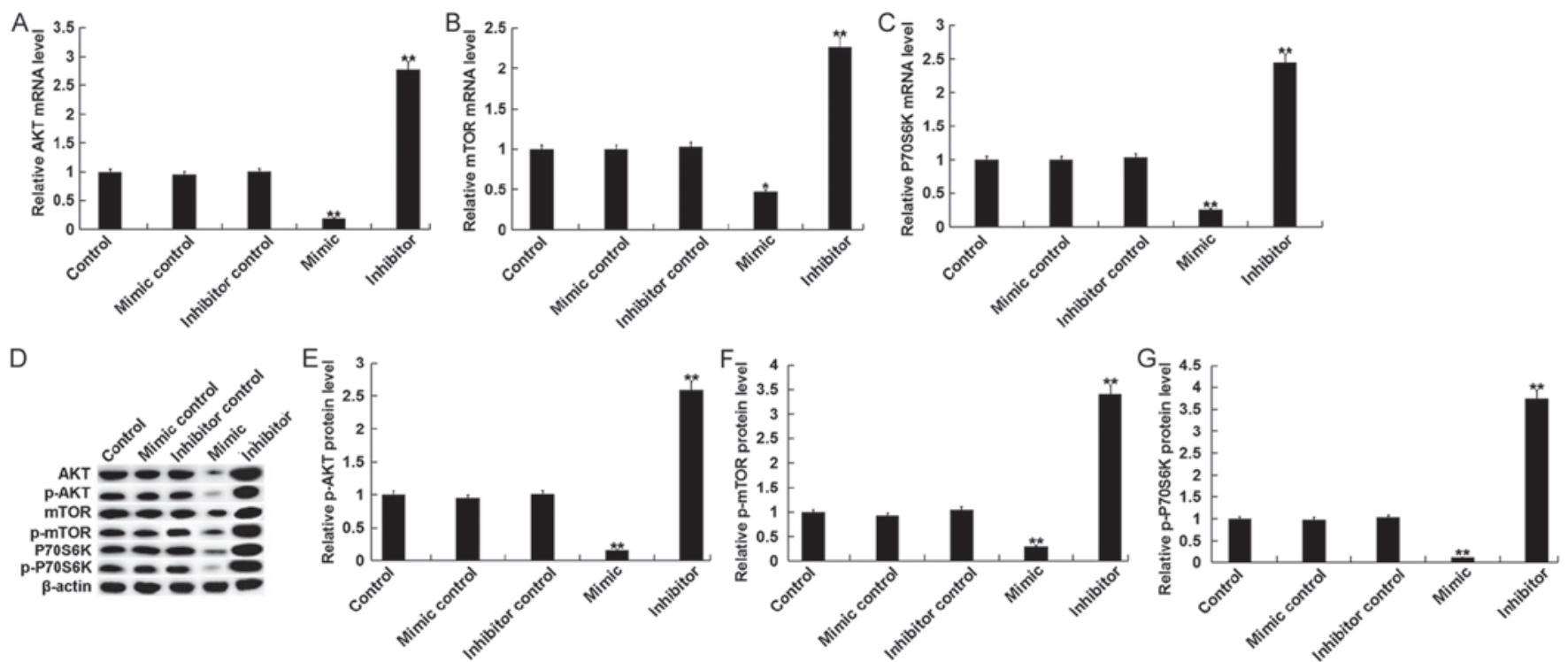

Figure 4. Effect of miR-15b on the PI3K/AKT signaling pathway in hVSMCs. Mimic controls, miR-15b mimics, miR-15b inhibitors or inhibitor controls were transfected into hVSMCs for $48 \mathrm{~h}$. Subsequently, mRNA levels of (A) AKT, (B) mTOR and (C) P70S6K, were assessed via reverse transcription-quantitative polymerase chain reaction. (D) Western blotting was also performed to assess the protein levels of AKT, p-AKT, mTOR, p-mTOR, P70S6K and p-P70S6K. (E) p-AKT, (F) p-mTOR and (G) p-P70S6K were quantified and presented as the fold of control. All data are presented as the mean \pm standard deviation. ${ }^{*} \mathrm{P}<0.05$ and ${ }^{* *} \mathrm{P}<0.01$ vs. the control. miR, microRNA; PI3K, phosphoinositide 3-kinase; AKT, protein kinase B; hVSMCs, human vascular smooth muscle cells; p, phosphorylated; mTOR, mechanistic target of rapamycin; P70S6K, ribosomal protein S6 kinase beta-1.

pathogenesis of atherosclerosis, its underlying pathophysiology remains unclear $(1,18,19)$. Previous studies have also identified the differential expression of certain genes in the peripheral monocytes of patients with a variety of atherosclerotic conditions (20-22). However, the association between PAD and certain miRNAs remains largely unclear (1).

miRNAs are small non-coding RNAs, which are involved in the regulation of multiple biological processes (1). They are considered to be part of a network in which a modest change in the expression of one miRNA may trigger a chain reaction involving multiple genes in the same or different pathways (23). It has been demonstrated that miRNAs can be used to diagnose
PAD and to further understand the molecular mechanism of disease development, which illustrates its potential to be a therapeutic target for PAD in the future (1).

miR-15b has been studied in a variety of diseases, particularly in cancer. Li and Wang (24) suggested that miR-15b may prevent amyloid- $\beta$ accumulation by targeting nuclear factor- $\kappa \mathrm{B}$ signaling and beta-secretase 1, thus exhibiting a protective effect in Alzheimer's disease. Furthermore, Sun et al (25) demonstrated that miR-15b levels were decreased in human gliomas, which was associated with a poor prognosis. Additionally, Wang et al (26) revealed that miR-15b facilitates non-small cell lung carcinoma cell proliferation and invasion 
A

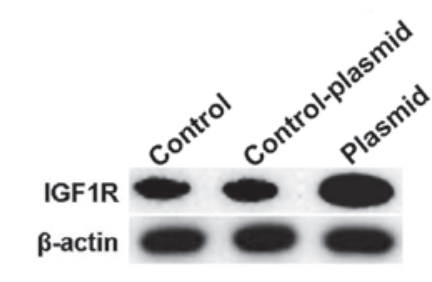

C

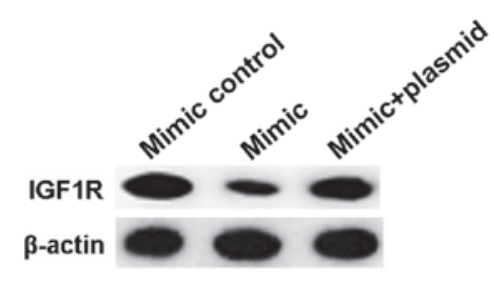

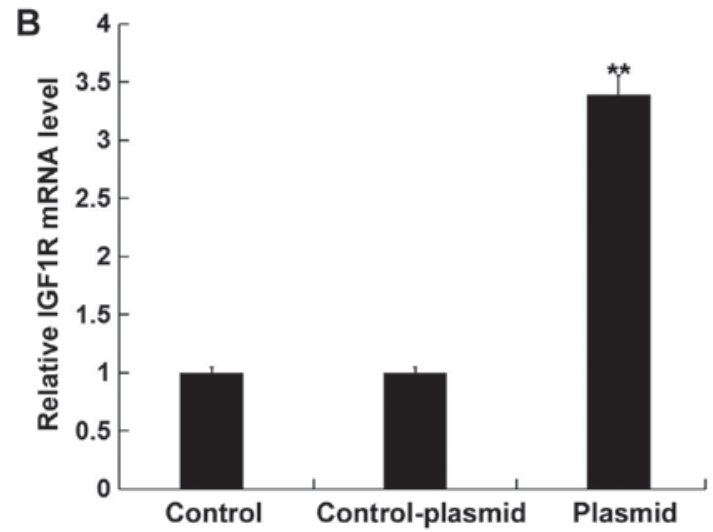

D

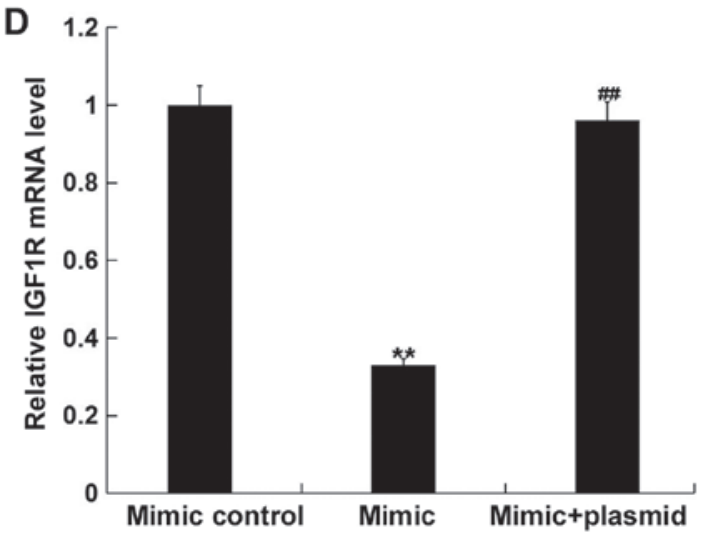

Figure 5. Effect of miR-15b on the expression of IGF1R in hVSMCs. hVSMCs were transfected with control-plasmids or IGF1R-plasmids for $48 \mathrm{~h}$. Subsequently, the (A) protein and (B) mRNA levels of IGF1R were determined via western blotting and RT-qPCR, respectively. hVSMCs were also transfected with mimic controls, miR-15b mimics or miR-15b mimic+IGF1R-plasmids for $48 \mathrm{~h}$, following which the (C) protein and (D) mRNA levels of IGF1R were determined via western blotting and RT-qPCR, respectively. All data are presented as the mean \pm standard deviation. ${ }^{* *} \mathrm{P}<0.01 \mathrm{vs}$. the control or mimic control; ${ }^{\# \prime} \mathrm{P}<0.01 \mathrm{vs}$. the mimic. miR, microRNA; IGF1R, insulin growth factor 1 receptor; hVSMCs, human vascular smooth muscle cells; RT-qPCR, reverse transcription-quantitative polymerase chain reaction.
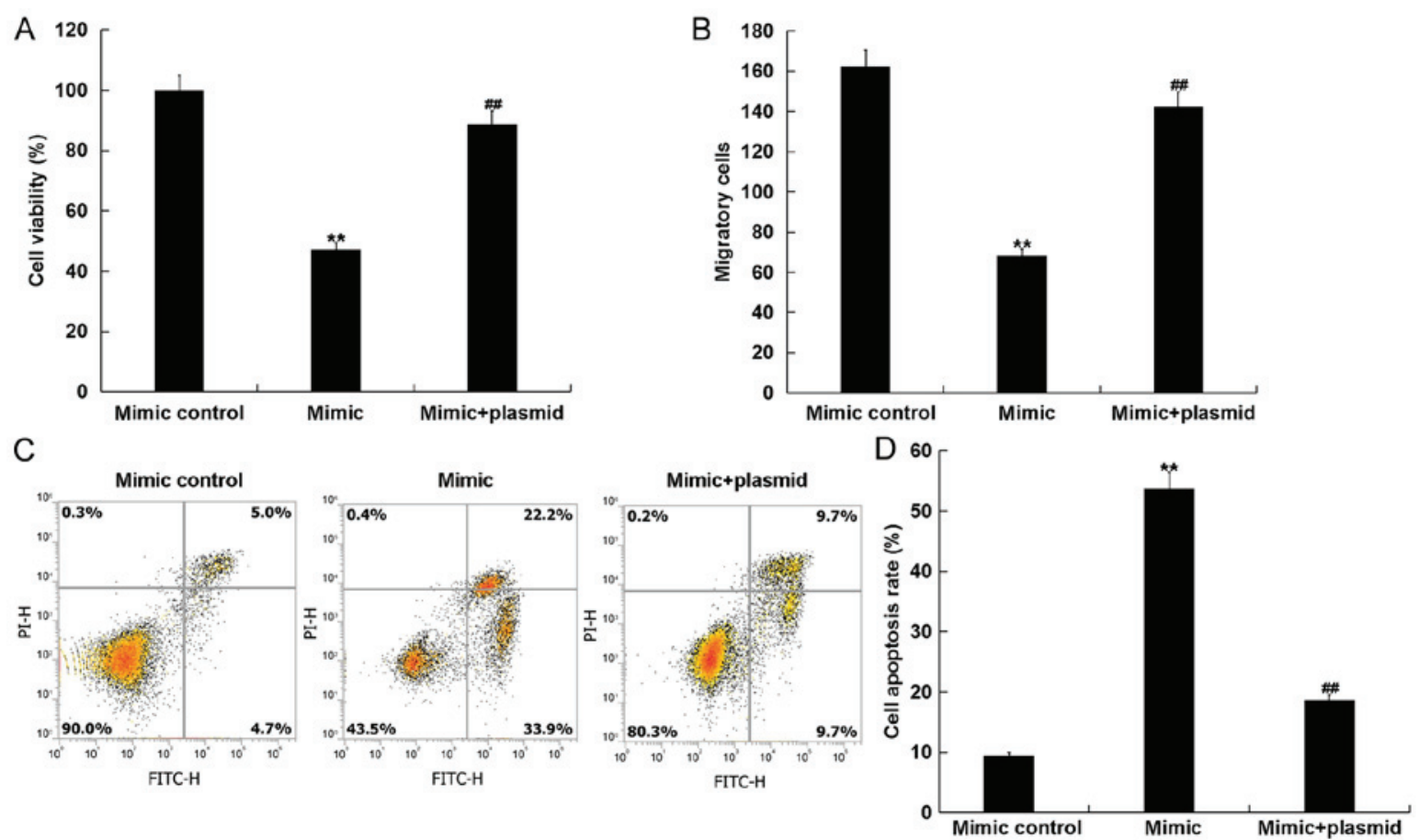

Figure 6. miR-15b inhibits hVSMC cell viability and migration, and induces cell apoptosis by directly targeting IGF1R. hVSMCs were transfected with mimic controls, miR-15b mimics or miR-15b mimic+IGF1R-plasmids for $48 \mathrm{~h}$. (A) Cell viability, (B) migration and (C) apoptosis with (D) quantification were assessed via MTT, transwell and flow cytometry assays, respectively. All data are presented as the mean \pm standard deviation. "* P $<0.01$ vs. the mimic control; ${ }^{\# \#} \mathrm{P}<0.01$ vs. the mimic. miR, microRNA; hVSMCs, human vascular smooth muscle cells; IGF1R, insulin growth factor 1 receptor; PI, propidium iodide; FITC, fluorescein isothiocyanate. 
by regulating the expression of metallopeptidase inhibitor 2 . miR-15b has also been revealed to contribute to extracellular matrix degradation in intervertebral disc degeneration by regulating the expression of SMAD family member 3 (27). However, the role of $\mathrm{miR}-15 \mathrm{~b}$ in PAD remains unclear. Therefore, the current study was performed to assess the role of miR-15b in the growth of hVSMCs.

To determine the role of miR-15b in the growth of hVSMCs, miR-15b was up- or downregulated in hVSMCs. Further analysis demonstrated that miR-15b overexpression significantly inhibited cell viability and migration, and induced apoptosis in hVSMCs, while miR-15b downregulation exhibited the opposite results. To further assess the molecular mechanism of miR-15b on hVSMCs, the targets of miR-15b were predicted using TargetScan software. The results revealed that IGF1R was a target of miR-15b that was negatively regulated by miR-15b in hVSMCs. IGF1R, as a transmembrane receptor tyrosine kinase, is an anti-apoptotic oncogene that is closely associated with the insulin receptor (InsR), which forms homodimers or heterodimerizes with InsR to discriminate its ligands (IGF-1 and IGF-2) (28). Upon binding to its ligand, IGF1R activates the PI3K/AKT and the mitogen activated protein kinase pathway (29). The current study also indicated that miR-15b overexpression repressed the PI3K/AKT pathway in hVSMCs, while miR-15b downregulation promoted PI3K/AKT pathway. Finally, to assess whether miR-15b affected the growth of hVSMCs by directly targeting IGF1R, a rescue experiment was performed. The inhibitory effect of miR-15b on the growth of hVSMCs was eliminated by IGF1R overexpression, indicating that miR-15b prevented the proliferation and migration, and induced the apoptosis of hVSMCs by directly targeting IGF1R.

In summary, the results of the current study indicated that miR-15b regulates cell proliferation, migration and apoptosis by regulating the PI3K/AKT signaling pathway via IGF1R targeting. Therefore, the exogenous overexpression of miR-15b may serve as a promising method for the treatment of PAD.

\section{Acknowledgements}

Not applicable.

\section{Funding}

No funding received.

\section{Availability of data and materials}

All data sets used and/or generated during the current study are available from the corresponding author on reasonable request.

\section{Authors' contributions}

YS collaborated to design the study. YS, YG, TS and CY were responsible for data collection and analysis. $\mathrm{ZN}$ and XW collaborated to data analysis. All authors collaborated to interpret results and develop the manuscript.

\section{Ethics approval and consent to participate}

Not applicable.

\section{Patient consent for publication}

Not applicable.

\section{Competing interests}

The authors declare that they have no competing interests.

\section{References}

1. Stather PW, Sylvius N, Wild JB, Choke E, Sayers RD and Bown MJ: Differential microRNA expression profiles in peripheral arterial disease. Circ Cardiovasc Genet 6: 490-497, 2013.

2. Togliatto G, Trombetta A, Dentelli P, Gallo S, Rosso A, Cotogni P, Granata R, Falcioni R, Delale T, Ghigo E and Brizzi MF: Unacylated ghrelin induces oxidative stress resistance in a glucose intolerance and peripheral artery disease mouse model by restoring endothelial cell miR-126 expression. Diabetes 64: 1370-1382, 2015

3. Chen L, Liu C, Sun D, Wang T, Zhao L, Chen W, Yuan M, Wang J and Lu W: MicroRNA-133a impairs perfusion recovery after hindlimb ischemia in diabetic mice. Biosci Rep 38: BSR20180346, 2018.

4. Hsu PY, Hsi E, Wang TM, Lin RT, Liao YC and Juo SH: MicroRNA let-7g possesses a therapeutic potential for peripheral artery disease. J Cell Mol Me 21: 519-529, 2017.

5. Dua A and Lee CJ: Epidemiology of peripheral arterial disease and critical limb ischemia. Tech Vasc Interv Radiol 19: 91-95, 2016.

6. Abu Dabrh AM, Steffen MW, Undavalli C, Asi N, Wang Z, Elamin MB, Conte MS and Murad MH: The natural history of untreated severe or critical limb ischemia. J Vasc Surg 62: 1642-1651.e3, 2015.

7. Rollins KE, Jackson D and Coughlin PA: Meta-analysis of contemporary short- and long-term mortality rates in patients diagnosed with critical leg ischaemia. Br J Surg 100: 1002-1008, 2013.

8. Mejias SG and Ramphul K: Prevalence of peripheral arterial disease among diabetic patients in Santo Domingo, Dominican Republic and associated risk factors. Arch Med Sci Atheroscler Dis 3: e35-e40, 2018.

9. Kloos W, Vogel B and Blessing E: MiRNAs in peripheral artery disease-something gripping this way comes. Vasa 43: 163-170, 2014.

10. Ganta VC, Choi MH, Kutateladze A, Fox TE, Farber CR and Annex BH: A MicroRNA93-interferon regulatory factor-9-immunoresponsive gene-1-itaconic acid pathway modulates M2-like macrophage polarization to revascularize ischemic muscle. Circulation 135: 2403-2425, 2017.

11. Fang J, Song XW, Tian J, Chen HY, Li DF, Wang JF, Ren AJ, Yuan WJ and Lin L: Overexpression of microRNA-378 attenuates ischemia-induced apoptosis by inhibiting caspase-3 expression in cardiac myocytes. Apoptosis 17: 410-423, 2012.

12. Zhou X, Yuan P and He Y: Role of microRNAs in peripheral artery disease (review). Mol Med Rep 6: 695-700, 2012.

13. Zampetaki A and Mayr M: MicroRNAs in vascular and metabolic disease. Circ Res 110: 508-522, 2012.

14. Shantikumar S, Caporali A and Emanueli C: Role of microRNAs in diabetes and its cardiovascular complications. Cardiovasc Res 93: 583-593, 2012.

15. Katare R, Riu F, Mitchell K, Gubernator M, Campagnolo P, Cui Y, Fortunato O, Avolio E, Cesselli D, Beltrami AP, et al: Transplantation of human pericyte progenitor cells improves the repair of infarcted heart through activation of an angiogenic program involving micro-RNA-132. Circ Res 109: 894-906, 2011.

16. Livak KJ and Schmittgen TD: Analysis of relative gene expression data using real-time quantitative PCR and the 2(-Delta Delta C(T)) method. Methods 25: 402-408, 2001.

17. Paraskevas KI, Kotsikoris I, Koupidis SA, Giannoukas AD and Mikhailidis DP: Ankle-brachial index: A marker of both peripheral arterial disease and systemic atherosclerosis as well as a predictor of vascular events. Angiology 61: 521-523, 2010.

18. Wu MY, Li CJ, Hou MF and Chu PY: New insights into the role of inflammation in the pathogenesis of atherosclerosis. Int $\mathrm{J}$ Mol Sci 18: E2034, 2017. 
19. Di Pietro N, Formoso G and Pandolfi A: Physiology and pathophysiology of oxLDL uptake by vascular wall cells in atherosclerosis. Vascul Pharmacol 84: 1-7, 2016.

20. Fu S, Zhao H, Shi J, Abzhanov A, Crawford K, Ohno-Machado L, Zhou J, Du Y, Kuo WP, Zhang J, et al: Peripheral arterial occlusive disease: Global gene expression analyses suggest a major role for immune and inflammatory responses. BMC Genomics 9: $369,2008$.

21. Wingrove JA, Daniels SE, Sehnert AJ, Tingley W, Elashoff MR, Rosenberg S, Buellesfeld L, Grube E, Newby LK, Ginsburg GS and Kraus WE: Correlation of peripheral-blood gene expression with the extent of coronary artery stenosis. Circ Cardiovase Genet 1: 31-38, 2008.

22. Masud R, Shameer K, Dhar A, Ding K and Kullo IJ: Gene expression profling of peripheral blood mononuclear cells in the setting of peripheral arterial disease. J Clin Bioinforma 2: 6, 2012.

23. Wang B, Hong W and Yang Z: MiR-122 inhibits cell proliferation and tumorigenesis of breast cancer by targeting IGF1R. PLoS One 7: e47053, 2012.

24. Li J and Wang H: miR-15b reduces amyloid- $\beta$ accumulation in SH-SY5Y cell line through targeting NF- $\kappa \mathrm{B}$ signaling and BACE1. Biosci Rep 38: BSR20180051, 2018.
25. Sun G, Yan S, Shi L, Wan Z, Jiang N, Li M and Guo J: Decreased expression of miR-15b in human gliomas is associated with poor prognosis. Cancer Biother Radiopharm 30: 169-173, 2015.

26. Wang $\mathrm{H}$, Zhan Y, Jin J, Zhang $\mathrm{C}$ and Li W: MicroRNA-15b promotes proliferation and invasion of non-small cell lung carcinoma cells by directly targeting TIMP2. Oncol Rep 37: 3305-3312, 2017.

27. Kang L, Yang C, Yin H, Zhao K, Liu W, Hua W, Wang K, Song Y, Tu J, Li S, et al: MicroRNA-15b silencing inhibits IL-1 $\beta$-induced extracellular matrix degradation by targeting SMAD3 in human nucleus pulposus cells. Biotechnol Lett 39: 623-632, 2017.

28. Solarek W, Czarnecka AM, Escudier B, Bielecka ZF, Lian F and Szczylik C: Insulin and IGFs in renal cancer risk and progression. Endocr Relat Cancer 22: R253-R264, 2015.

29. Pollak M: The insulin and insulin-like growth factor receptor family in neoplasia: An update. Nat Rev Cancer 12: 159-169, 2012.

This work is licensed under a Creative Commons Attribution-NonCommercial-NoDerivatives 4.0 International (CC BY-NC-ND 4.0) License. 